\title{
Right ventricular dyssynchrony and exercise capacity in idiopathic pulmonary arterial hypertension
}

\author{
Roberto Badagliacca ${ }^{1}$, Silvia Papa ${ }^{1}$, Gabriele Valli ${ }^{1}$, Beatrice Pezzuto ${ }^{1}$, \\ Roberto Poscia (101, Manuela Reali ${ }^{1}$, Giovanna Manzi ${ }^{1}$, Elisa Giannetta ${ }^{2}$, \\ Daniele Berardi ${ }^{1}$, Susanna Sciomer ${ }^{1}$, Paolo Palange ${ }^{3}$, Francesco Fedele ${ }^{1}$, \\ Robert Naeije ${ }^{4}$ and Carmine Dario Vizza ${ }^{1}$
}

Affiliations: ${ }^{1}$ Dept of Cardiovascular and Respiratory Science, Sapienza University of Rome, Rome, Italy. ${ }^{2}$ Dept of Experimental Medicine, Sapienza University of Rome, Rome, Italy. ${ }^{3}$ Dept of Clinical Medicine, Sapienza University of Rome, Rome, Italy. ${ }^{4}$ Dept of Cardiology, Erasme University Hospital, Brussels, Belgium.

Correspondence: Roberto Badagliacca, Department of Cardiovascular and Respiratory Science, I School of Medicine, Sapienza University of Rome, Policlinico Umberto I, Viale del Policlinico 155 - 00161 Rome, Italy. E-mail: roberto.badagliaccaQuniroma1.it

@ERSpublications

Speckle tracking assessment of RV dyssynchrony improves the prediction of aerobic exercise capacity in IPAH http://ow.ly/yHyh30bedtD

Cite this article as: Badagliacca R, Papa S, Valli G, et al. Right ventricular dyssynchrony and exercise capacity in idiopathic pulmonary arterial hypertension. Eur Respir J 2017; 49: 1601419 [https://doi.org/ 10.1183/13993003.01419-2016].

ABSTRACT Survival in patients with pulmonary arterial hypertension (PAH) is determined by right ventricular (RV) function adaptation to afterload. How altered RV function impacts on exercise capacity in $\mathrm{PAH}$ is not exactly known.

104 idiopathic PAH (IPAH) patients aged 52 \pm 14 years underwent a diagnostic right heart catheterisation, a comprehensive echocardiography including two-dimensional speckle tracking for RV dyssynchrony evaluation and a cardiopulmonary exercise test. Multivariate analyses were performed to identify independent predictors of peak oxygen uptake (peak $V^{\prime} \mathrm{O}_{2}$ ).

A first multivariate analysis of only resting haemodynamic variables identified cardiac index, right atrial (RA) pressure and pulmonary arterial compliance as independent predictors, with low predictive capacity $\left(\mathrm{r}^{2}=0.31 ; \mathrm{p}<0.001\right)$. A second multivariate analysis model which considered only echocardiographic parameters but without RV dyssynchrony, identified RV fractional area change (FAC) and RA area as independent predictors with still low predictivity $\left(\mathrm{r}^{2}=0.35 ; \mathrm{p}<0.001\right)$. Adding RV dyssynchrony to the second model increased its predictivity $\left(\mathrm{r}^{2}=0.48 ; \mathrm{p}<0.001\right)$. Repetition of the three multivariate analyses in patients with preserved RVFAC confirmed that inclusion of RV dyssynchrony results in the highest predictive capability of peak $V^{\prime} \mathrm{O}_{2}\left(\mathrm{r}^{2}=0.53 ; \mathrm{p}=0.001\right)$.

A comprehensive echocardiography with speckle tracking-derived assessment of the heterogeneity of RV contraction improves the prediction of aerobic exercise capacity in IPAH.

Received: July 152016 | Accepted after revision: March 072017

Conflict of interest: Disclosures can be found alongside this article at erj.ersjournals.com

Copyright @ERS 2017 


\section{Introduction}

It has been better realised in recent years that symptomatology, functional state, exercise capacity and survival in pulmonary arterial hypertensions (PAH) are essentially to be accounted for by right ventricular (RV) function [1, 2]. Studies relying on pressure-volume loops measured by high-fidelity manometer-tipped catheters and advanced imaging with cardiac magnetic resonance (CMR) have shown that coupling of RV function to the increased resistive state of the pulmonary circulation basically relies on an increased contractility (or end-systolic elastance, Ees) to match the increased afterload (or arterial elastance, Ea) [2,3]. However, bedside assessment of RV arterial coupling with Ees and Ea determined by high-fidelity catheters and CMR is not possible in daily clinical practice. A number of simpler surrogate right heart catheterisation- and echocardiography-derived parameters of RV function have been shown to be of prognostic relevance [3], but the independent impact of each of them on symptomatology and exercise capacity has not been exactly defined.

Standard echocardiography in patients with pulmonary hypertension includes estimates of pulmonary artery pressure (PAP) combined with measurements of RV structure and global function [4]. However, recent imaging by tissue Doppler, magnetic resonance imaging (MRI) or speckle tracking echocardiography have shown that increased PAP may be associated with a considerable heterogeneity of RV regional function, or dyssynchrony, prolonged contraction and eventual post-systolic shortening [512]. Although RV dyssychrony has been shown to be an independent predictor of disease severity and outcome in PAH $[11,12]$, how it affects cardiac output adaptation to metabolic demand during exercise has not yet been explored.

We hypothesised that RV dyssychrony would decrease the efficiency of RV contraction and thereby decrease exercise capacity. Therefore, the aim of this study was to analyse the determinants of exercise capacity as defined by peak oxygen uptake (peak $V^{\prime} \mathrm{O}_{2}$ ) during a cardiopulmonary exercise test (CPET) among right heart catheterisation and standard echocardiographic measurements with and without assessment of RV dyssynchrony.

\section{Methods}

\section{Study population}

108 consecutive patients with idiopathic PAH gave informed consent to the study, which was approved by the Institutional Review Board for human studies of the Policlinico Umberto I - Sapienza University of Rome (Protocol no. 42412). Four patients were excluded because of poor echocardiographic imaging quality. Thus, 104 idiopathic PAH patients were enrolled in the study, 65 women and 39 men, aged 52 \pm 14 years, with a body mass index of $25 \pm 4 \mathrm{~kg} \cdot \mathrm{m}^{-2}$, a WHO functional class of $2.8 \pm 0.4$ and a 6 -min walk distance of $430 \pm 60 \mathrm{~m}$. The diagnosis of idiopathic PAH rested on dyspnoea-fatigue symptomatology, right heart catheterisation showing a mean PAP (mPAP) $\geqslant 25 \mathrm{mmHg}$ and a wedged PAP (PAWP) $\leqslant 15 \mathrm{mmHg}$, and a step-by-step approach including lung function tests, a computed tomography scan of the chest, a ventilation/perfusion scan, echocardiography and laboratory tests to exclude left heart conditions with increased pulmonary venous pressure, lung diseases, thromboembolism, connective tissue disease and other associated PAH conditions, following recently updated guidelines [13].

\section{Right heart catheterisation}

The patients underwent right heart catheterisation with a triple lumen balloon-tipped thermodilution Swan-Ganz catheter in the supine position with zero at the mid-chest level and pressures measured at end-expiration. The measurements included PAP, PAWP, right atrial pressure (RAP) and cardiac output (CO). Pulmonary vascular resistance (PVR) was calculated as (mPAP - PAWP)/CO, and PA compliance as (systolicPAP - diastolicPAP)/stroke volume.

\section{Echocardiographic assessment}

A comprehensive echocardiography was performed within $24 \mathrm{~h}$ after the right heart catheterisation. All echocardiographic data were acquired by the same operator using commercially available equipment (Vivid S6, GE), with a $3.5-\mathrm{MHz}$ transducer at a depth of $16 \mathrm{~cm}$ in the standard views; the patient was in the left lateral decubitus position. Standard M-mode, 2D and Doppler images were obtained during breath-hold at end expiration and measurements were obtained from the mean of three consecutive beats in accordance with the American Society of Echocardiography Guidelines [4]. The following standard parameters and derived measures were considered in the analysis: right atrial (RA) area, RV end-diastolic area (RVEDA), RV end-systolic area (RVESA), RV fractional area change $\%($ RVFAC $=($ RVEDA-RVESA $) /$ RVEDA $\times 100)$, tricuspid annular plane systolic excursion (TAPSE), left ventricular systolic and diastolic eccentricity index (LV-EIs and LV-EId, respectively) and presence of pericardial effusion. Tricuspid regurgitation was semiquantitatively graded considering the regurgitant jet area using colour Doppler imaging. The transmitral flow velocity curve was obtained using pulsed Doppler 
imaging, positioning the sample volume between the tips of the mitral leaflets. E- and A-wave peak velocities and the ratio of early transmitral flow velocity to atrial flow velocity were measured.

\section{D Speckle tracking echocardiography}

Acquisition

For speckle tracking analysis (EchoPAC workstation 7.0.1, GE Medical Systems), standard greyscale 2D images in the RV dedicated four-chamber apical view were acquired (frame rate $>60 \mathrm{fps}$ ) and digitally stored in a 3 beats cine-loop format.

\section{Analysis}

To assess the segmental characteristics of the RV, we adopted the six-segment model, excluding the apical segments for the analysis because of the high variability of these segments observed even in normal subjects, as previously reported and discussed [11, 12]. For the quantification of RV dyssynchrony we considered longitudinal strain and calculated the standard deviation of the times to peak-systolic strain for the four mid-basal RV segments corrected to the R-R interval between two QRS complexes, according to the Bazett's formula, and called RV-SD4. Using the upper 95\% limit of normal (mean+2 SD) of healthy subjects previously reported by our group [12], we defined a cut-off value of $18 \mathrm{~ms}$ as the criterion for RV dyssynchrony.

For RV-SD4 measurement, intra-observer and inter-observer variability were assessed for 20 randomly selected patients by the Bland-Altman method, with results similar to previously published data [11, 12]: 0.00 \pm 2.05 (95\% CI -3.94 to 3.94 ) (average percentage variability $6.4 \%$ ) and $-0.37 \pm 1.89$ (95\% CI -4.08 to 3.34 ) (average percentage variability 5.9\%), respectively, which can be considered acceptable for our clinical purpose.

\section{Cardiopulmonary exercise test}

All patients performed a symptom-limited incremental cycler ergometer CPET with $10-15 \mathrm{~W} \cdot \mathrm{min}^{-1}$ workload increments. Oxygen uptake $\left(V^{\prime} \mathrm{O}_{2}\right)$, carbon dioxide output $\left(V^{\prime} \mathrm{CO}_{2}\right)$ and minute ventilation $\left(V^{\prime} \mathrm{E}\right)$ were measured breath-by-breath (Quark CPET, Rome, Italy) and averaged every $5 \mathrm{~s}$ for subsequent analysis. Oxygen pulse was calculated as $V^{\prime} \mathrm{O}_{2} / \mathrm{HR}$. Heart rate (HR) was monitored via a 12-lead ECG. Tests were considered maximal if the peak respiratory exchange ratio (RER) was greater than 1.1.

\section{Statistical analysis}

Continuous data were expressed as mean \pm standard deviation, and categorical data were expressed as counts and proportions. Two-group comparisons were done with unpaired, two-tailed t-tests for means if the data were normally distributed or with Wilcoxon's rank-sum tests if the data were not normally distributed. Chi-squared or Fisher's exact tests were used to analyse the categorical data.

Linear regression analysis was performed to assess the relations between RVFAC and peak $V^{\prime} \mathrm{O}_{2}$ and expressed as a Pearson correlation coefficient. Patients were further divided according to the tertile of RV dyssynchrony to build a scatterplot.

The median value of RVFAC (38\%) was used to divide the population into two groups, low and high systolic function, as this value resembles the lower limit of normal suggested by guidelines [4].

Multivariate regression analysis was used to identify the variables that were associated with peak $V^{\prime} \mathrm{O}_{2}$ by a stepwise variable selection method with significance level to entry 0.1 and significance level to stay 0.05 . Three models were constructed for the overall population: Model-1 was limited to haemodynamic variables, Model-2 was limited to echocardiographic variables excluding RV dyssynchrony, Model-3 was limited to echocardiographic variables including RV dyssynchrony. Another six models were constructed for subgroups of patients with RVFAC above and below 38\%: Model-4 and Model-7 were limited to haemodynamic variables; Model-5 and Model-8 were limited to echocardiographic variables excluding RV dyssynchrony; Model-6 and Model-9 were limited to echocardiographic variables including RV dyssynchrony. Predictive accuracy of the models was compared using $\mathrm{r}^{2}$, and details for each model are reported, including corresponding $\mathrm{r}^{2}$, $\mathrm{p}$-value, constant, and regression coefficient.

All statistical analyses were performed using SPSS software (version 20.0, IBM) and Stata 13 (StataCorp, College Station, TX, USA). All statistical tests were two-sided, and $\mathrm{p}<0.05$ was considered statistically significant.

\section{Results}

As shown in table 1, patients presented with a haemodynamic profile compatible with severe pulmonary hypertension, echocardiography showing increased RV dimensions and depressed systolic function and a CPET showing markedly decreased peak $V^{\prime} \mathrm{O}_{2}$, workload, $\mathrm{O}_{2}$ pulse and maximum $\mathrm{HR}$ with increased 
TABLE 1 Haemodynamic, imaging and exercise characteristics of the study population

\begin{tabular}{|c|c|}
\hline \multicolumn{2}{|l|}{ Haemodynamics } \\
\hline mPAP $\mathrm{mmHg}$ & $48 \pm 15$ \\
\hline $\mathrm{RAP} \mathrm{mmHg}$ & $7 \pm 4$ \\
\hline $\mathrm{Cl} \mathrm{L} \cdot \mathrm{min}^{-1} \cdot \mathrm{m}^{-2}$ & $2.4 \pm 0.4$ \\
\hline PAWP mmHg & $9 \pm 3$ \\
\hline PVR Wood units & $9.7 \pm 5.1$ \\
\hline Compliance $\mathrm{mL} \cdot \mathrm{mmHg}^{-1}$ & $1.5 \pm 0.8$ \\
\hline \multicolumn{2}{|l|}{ Echocardiography } \\
\hline $\mathrm{RA}$ area $\mathrm{cm}^{2}$ & $31 \pm 10$ \\
\hline LV-Eld & $1.40 \pm 0.32$ \\
\hline LV-Els & $1.60 \pm 0.61$ \\
\hline RVEDA $\mathrm{cm}^{2}$ & $27.5 \pm 7.4$ \\
\hline RVESA $\mathrm{cm}^{2}$ & $17.2 \pm 6.0$ \\
\hline RVFAC $\%$ & $37.9 \pm 8.6$ \\
\hline TAPSE $\mathrm{mm}$ & $19.6 \pm 3.8$ \\
\hline Pericardial effusion & $16(15 \%)$ \\
\hline Peak 2DS basal RVFW \% & $21 \pm 6$ \\
\hline Peak 2DS mid RVFW \% & $19 \pm 7$ \\
\hline RV dyssynchrony ms & $30 \pm 24$ \\
\hline \multicolumn{2}{|c|}{ Cardiopulmonary exercise test } \\
\hline HR rest beats $\cdot \min ^{-1}$ & $81 \pm 14$ \\
\hline HR peak beats $\cdot \min ^{-1}$ & $128 \pm 24$ \\
\hline$V^{\prime} \mathrm{O}_{2}$ peak $\mathrm{mL} \cdot \mathrm{kg}^{-1} \cdot \mathrm{min}^{-1}$ & $15.4 \pm 4.1$ \\
\hline $\mathrm{O}_{2}$ pulse peak $\mathrm{mL}$ & $8.3 \pm 2.7$ \\
\hline$V^{\prime} \mathrm{E} / V^{\prime} \mathrm{CO}_{2}$ slope & $47 \pm 13$ \\
\hline Work peak W & $66 \pm 30$ \\
\hline
\end{tabular}

mPAP: mean pulmonary arterial pressure; RAP: mean right atrial pressure; $\mathrm{Cl}$ : cardiac index; PAWP: mean pulmonary arterial wedged pressure; PVR: pulmonary vascular resistance; RA area: right atrium area; LV-Eld: left ventricular end-diastolic eccentricity index; LV-Els: left ventricular end-systolic eccentricity index; RVEDA: right ventricular end-diastolic area; RVESA: right ventricular end-systolic area; RVFAC: right ventricular fractional area change; TAPSE: tricuspid annular plane systolic excursion; peak 2DS mid RVFW: two-dimensional strain of the mid segment of the right ventricular free wall by Speckle Tracking Echocardiography; peak 2DS basal RVFW: two-dimensional strain of the basal segment of the right ventricular free wall by Speckle Tracking Echocardiography; RV: right ventricular; HR: heart rate; $V^{\prime} \mathrm{O}_{2}$ : oxygen uptake; $V^{\prime} E / V^{\prime} \mathrm{CO}_{2}$ slope: ventilation to $\mathrm{CO}_{2}$ production slope.

$V^{\prime} \mathrm{E} / V^{\prime} \mathrm{CO}_{2}$ slope. There was marked RV dyssynchrony, with RV-SD4 values ranging from 0 to $124 \mathrm{~ms}$, and exceeding $18 \mathrm{~ms}$ in $60 \%$ of the patients.

There was a significant correlation between RVFAC and peak $V^{\prime} \mathrm{O}_{2} \quad\left(\mathrm{r}^{2}=0.39 ; \mathrm{p}=0.0001\right)$. The correlation between TAPSE and peak $V^{\prime} \mathrm{O}_{2}$ was also significant, but weaker $\left(\mathrm{r}^{2}=0.18 ; \mathrm{p}<0.001\right)$.

Analysing the characteristics of the two groups of patients based on the median value of RVFAC (38\%) (table 2), those $\leqslant 38 \%$ had a worse WHO functional class, shorter 6-min walk distance, worse haemodynamics and more advanced RV remodelling and dyssynchrony. Considering the CPET, all patients reached maximal exercise from a metabolic point of view (peak respiratory exchange ratio $>1.1$ in both groups). Patients with RVFAC $\leqslant 38 \%$ had lower peak workload, $V^{\prime} \mathrm{O}_{2}$ and $\mathrm{O}_{2}$ pulse, and reduced ventilatory efficiency (higher $V^{\prime} \mathrm{E} / V^{\prime} \mathrm{CO}_{2}$ slope).

In the overall population (table 3), a first multivariate analysis model considering resting hemodynamic variables exclusively identified CI, RAP and pulmonary artery compliance as independent predictors of peak $V^{\prime} \mathrm{O}_{2}$, but with low predictive capability (Model-1, $\mathrm{r}^{2}=0.31 ; \mathrm{p}<0.001$ ). A second model considering only echocardiographic variables (RVEDA, RVESA, RVFAC, TAPSE, RA area, pericardial effusion, LV-EId and LV-EIs) without RV dyssynchrony evaluation disclosed RVFAC and RA area as independent predictors, but with low predictive capability (Model-2, $\mathrm{r}^{2}=0.35 ; \mathrm{p}<0.001$ ). A third model with RV dyssynchrony added to echocardiographic variables disclosed RVFAC, RA area and RV dyssynchrony as independent predictors, with much stronger predictive capability (Model-3, $\mathrm{r}^{2}=0.48 ; \mathrm{p}<0.001$ ).

In the group of patients with preserved RVFAC (table 4), RAP and pulmonary artery compliance emerged as the independent factors in the haemodynamic model (Model-4, $\mathrm{r}^{2}=0.18 ; \mathrm{p}<0.01$ ) and confirmed a low predictive capability of peak $V^{\prime} \mathrm{O}_{2}$ from haemodynamic measurements. Among the echocardiographic parameters excluding RV dyssynchrony evaluation, RVEDA and RA area remained independent predictors of peak $V^{\prime} \mathrm{O}_{2}$ (Model-5, $\left.\mathrm{r}^{2}=0.21 ; \mathrm{p}<0.01\right)$. Adding $\mathrm{RV}$ dyssynchrony to the echocardiographic evaluation a 
TABLE 2 Demographic, clinical, haemodynamic, imaging and exercise differences in the two groups of patients based on the median value of right ventricular fractional area change (RVFAC)

\begin{tabular}{|c|c|c|c|}
\hline & RVFAC $\leqslant 38 \%$ & RVFAC $>38 \%$ & p-value \\
\hline Patients $\mathrm{n}$ & 52 & 52 & \\
\hline Age years & $53 \pm 14$ & $52 \pm 13$ & NS \\
\hline $\mathrm{BMI}$ & $25.8 \pm 5$ & $24.9 \pm 4$ & NS \\
\hline WHO class & $3.0 \pm 0.2$ & $2.6 \pm 0.5$ & 0.0001 \\
\hline 6MWT m & $397 \pm 57$ & $452 \pm 60$ & 0.0001 \\
\hline \multicolumn{4}{|l|}{ Haemodynamics } \\
\hline $\mathrm{mPAP} \mathrm{mmHg}$ & $57 \pm 16$ & $42 \pm 12$ & 0.0001 \\
\hline $\mathrm{RAP} \mathrm{mmHg}$ & $9.2 \pm 4.9$ & $6.3 \pm 2.8$ & 0.0001 \\
\hline $\mathrm{CI} L \cdot \mathrm{min}^{-1} \cdot \mathrm{m}^{-2}$ & $2.2 \pm 0.4$ & $2.7 \pm 0.3$ & 0.0001 \\
\hline PAWP mmHg & $8.9 \pm 3.2$ & $9.5 \pm 3.0$ & NS \\
\hline PVR Wood units & $13.4 \pm 5.3$ & $7.2 \pm 3.1$ & 0.0001 \\
\hline Compliance $\mathrm{mL} \cdot \mathrm{mmHg}^{-1}$ & $1.03 \pm 0.45$ & $1.84 \pm 0.79$ & 0.0001 \\
\hline \multicolumn{4}{|l|}{ Echocardiography } \\
\hline $\mathrm{RA}$ area $\mathrm{cm}^{2}$ & $34 \pm 10$ & $28 \pm 9$ & 0.002 \\
\hline LV-Eld & $1.58 \pm 0.35$ & $1.28 \pm 0.22$ & 0.0001 \\
\hline LV-Els & $1.87 \pm 0.84$ & $1.42 \pm 0.28$ & 0.0001 \\
\hline RVEDA $\mathrm{cm}^{2}$ & $31.6 \pm 7.1$ & $24.7 \pm 6.1$ & 0.0001 \\
\hline RVESA $\mathrm{cm}^{2}$ & $22.0 \pm 5.0$ & $13.9 \pm 3.9$ & 0.0001 \\
\hline TAPSE mm & $17.6 \pm 3.8$ & $20.8 \pm 3.1$ & 0.0001 \\
\hline Pericardial effusion & $5(9.6 \%)$ & $11(21.1 \%)$ & 0.01 \\
\hline Peak 2DS basal RVFW \% & $19 \pm 6$ & $24 \pm 5$ & 0.01 \\
\hline Peak 2DS mid RVFW \% & $16 \pm 7$ & $22 \pm 6$ & 0.001 \\
\hline RV dyssynchrony ms & $44.6 \pm 26.3$ & $20.9 \pm 16.9$ & 0.0001 \\
\hline \multicolumn{4}{|l|}{ Cardiopulmonary exercise test } \\
\hline HR rest beats & $82 \pm 15$ & $80 \pm 14$ & NS \\
\hline HR peak beats & $123 \pm 27$ & $132 \pm 21$ & NS \\
\hline$V^{\prime} \mathrm{O}_{2}$ peak $\mathrm{mL} \cdot \mathrm{kg}^{-1} \cdot \mathrm{min}^{-1}$ & $12.8 \pm 2.5$ & $17.2 \pm 4.0$ & 0.0001 \\
\hline$V^{\prime} \mathrm{O}_{2}$ pulse peak $\mathrm{mL} \cdot$ beat $^{-1}$ & $6.8 \pm 1.5$ & $9.3 \pm 2.8$ & 0.0001 \\
\hline$V^{\prime} E / V^{\prime} \mathrm{CO}_{2}$ slope & $50 \pm 14$ & $45 \pm 12$ & 0.001 \\
\hline
\end{tabular}

BMI: body mass index; WHO: World Health Organization; 6MWT: 6-min walk test; mPAP: mean pulmonary arterial pressure; RAP: mean right atrial pressure; $\mathrm{Cl}$ : cardiac index; PAWP: mean pulmonary arterial wedge pressure; PVR: pulmonary vascular resistance; RA area: right atrium area; LV-Eld: left ventricular end-diastolic eccentricity index; LVEIs: left ventricular end-systolic eccentricity index; RVEDA: right ventricular end-diastolic area; RVESA: right ventricular end-systolic area; TAPSE: tricuspid annular plane systolic excursion; peak 2DS mid RVFW: two-dimensional strain of the mid segment of the right ventricular free wall by Speckle Tracking Echocardiography; peak 2DS basal RVFW: two-dimensional strain of the basal segment of the right ventricular free wall by Speckle Tracking Echocardiography; RV: right ventricular; $\mathrm{HR}$ : heart rate; $V^{\prime} \mathrm{O}_{2}$ : oxygen uptake; $V^{\prime} \mathrm{E} / \mathrm{V}^{\prime} \mathrm{CO}_{2}$ slope: ventilation to $\mathrm{CO}_{2}$ production slope.

final model was generated, in which the only independent variables remaining were RA area and RV dyssynchrony, but with incremental predictive capability compared with all the other models (Model-6, $\left.\mathrm{r}^{2}=0.53 ; \mathrm{p}<0.001\right)$.

A scatterplot of RVFAC versus peak $V^{\prime} \mathrm{O}_{2}$ (figures 1 and 2) highlights how the presence of RV dyssynchrony may play a role among those patients with low peak $V^{\prime} \mathrm{O}_{2}$ and preserved systolic function, as documented by regression modelling analysis. As shown in figure 1, patients with the intermediate and the upper tertile of $\mathrm{RV}$ dyssynchrony distribution are more common among the group of patients with low peak $V^{\prime} \mathrm{O}_{2}$ and preserved systolic function (lower-right quadrant) than those with high peak $V^{\prime} \mathrm{O}_{2}$ and preserved systolic function (higher-right quadrant).

Indeed, the upper and intermediate tertiles of RV dyssynchrony distribution were associated with a more impaired WHO functional class, exercise capacity, haemodynamic condition and RV sizes and systolic function than the lower tertile (table 5, figure 3).

Finally, in the group of patients with RVFAC $\leqslant 38 \%$ (table 6), CI and RAP emerged as independent variables in the haemodynamic model (Model-7, $\mathrm{r}^{2}=0.48 ; \mathrm{p}<0.001$ ) confirming the high predictive capability of peak $V^{\prime} \mathrm{O}_{2}$ in the subset of patients with more impaired RV dysfunction. Among the echocardiographic parameters excluding RV dyssynchrony, RVEDA and LV-EId remained independent predictors of peak $V^{\prime} \mathrm{O}_{2}$ (Model-8, $\left.\mathrm{r}^{2}=0.26 ; \mathrm{p}<0.01\right)$. Adding $\mathrm{RV}$ dyssynchrony to the echocardiographic 


\begin{tabular}{|c|c|c|c|c|c|}
\hline & B & SE & Beta & p-value & $r^{2}$ \\
\hline Haemodynamic model & & & & & 0.31 \\
\hline Intercept & 10.12 & 2.40 & & $<0.001$ & \\
\hline $\mathrm{Cl}$ & 2.32 & 0.97 & 0.25 & 0.019 & \\
\hline RAP & -0.25 & 0.09 & -0.25 & 0.009 & \\
\hline Compliance & 1.13 & 0.55 & 0.21 & 0.043 & \\
\hline Echo model without RV dyssynchrony & & & & & 0.35 \\
\hline Intercept & 8.42 & 2.04 & & $<0.001$ & \\
\hline RVFAC & 0.27 & 0.03 & 0.54 & $<0.001$ & \\
\hline RA area & -0.10 & 0.03 & -0.25 & 0.002 & \\
\hline Echo model with RV dyssynchrony & & & & & 0.48 \\
\hline Intercept & 11.7 & 2.40 & & $<0.001$ & \\
\hline RVFAC & 0.20 & 0.04 & 0.42 & $<0.001$ & \\
\hline RA area & -0.10 & 0.03 & -0.24 & 0.002 & \\
\hline RV dyssynchrony & -0.04 & 0.01 & -0.22 & 0.01 & \\
\hline
\end{tabular}

$\mathrm{Cl}$ : cardiac index; RAP: mean right atrial pressure; RV: right ventricular; RVFAC: right ventricular fractional area change; RA area: right atrium area.

evaluation, the RVEDA and LV-EId remained independent factors associated with RV dyssynchrony, presenting incremental predictive capability compared with Model-8 (Model-9, $\mathrm{r}^{2}=0.46 ; \mathrm{p}<0.001$ ). Interestingly, the later model was not able to improve peak $V^{\prime} \mathrm{O}_{2}$ predictive capability compared with the haemodynamic model.

\section{Discussion}

The present results show that echocardiographic imaging of RV function with speckle tracking quantification of regional heterogeneity of contraction is of added value with respect to right heart catheterisation-derived indices to account for decreased aerobic exercise capacity in patients with idiopathic PAH.

Exercise capacity is impaired in $\mathrm{PAH}$, with typically a marked decrease in peak $V^{\prime} \mathrm{O}_{2}$ and $\mathrm{O}_{2}$ pulse and enhanced ventilatory responses [14]. This is thought to be due to a decreased cardiac output response to increased metabolic demand, with the addition of increased ventilation explaining exercise-induced dyspnoea and fatigue. Decreased maximum cardiac output in severe pulmonary hyptertension is essentially explained by the uncoupling of RV systolic function to the pulmonary circulation (i.e. impaired homeometric adaptation) [15] with eventual compensation by increased RV dimensions (heterometric adaptation) $[16,17]$. Increased RA and RV surface areas, increased eccentricity indices and depressed FAC

TABLE 4 Multivariate analysis of echocardiographic and haemodynamic variables associated with peak oxygen uptake in the subgroup of patients with right ventricular fractional area change $>38 \%$

\begin{tabular}{|c|c|c|c|c|c|}
\hline & B & SE & Beta & p-value & $r^{2}$ \\
\hline Haemodynamic model & & & & & 0.18 \\
\hline Intercept & 8.14 & 1.50 & & $<0.001$ & \\
\hline RAP & -0.34 & 0.18 & -0.24 & 0.05 & \\
\hline Compliance & 1.06 & 0.14 & 0.29 & 0.04 & \\
\hline Echo model without RV dyssynchrony & & & & & 0.21 \\
\hline Intercept & 23.7 & 2.21 & & $<0.001$ & \\
\hline RVEDA & -0.25 & 0.08 & -0.38 & 0.006 & \\
\hline RA area & -0.15 & 0.05 & -0.36 & 0.003 & \\
\hline Echo model with RV dyssynchrony & & & & & 0.53 \\
\hline Intercept & 12.6 & 0.51 & & $<0.001$ & \\
\hline RA area & -0.15 & 0.05 & -0.36 & 0.006 & \\
\hline RV dyssynchrony & -0.16 & 0.02 & -0.73 & $<0.001$ & \\
\hline
\end{tabular}

RAP: mean right atrial pressure; RV: right ventricular; RVEDA: right ventricular end-diastolic area; RA area: right atrium area. 


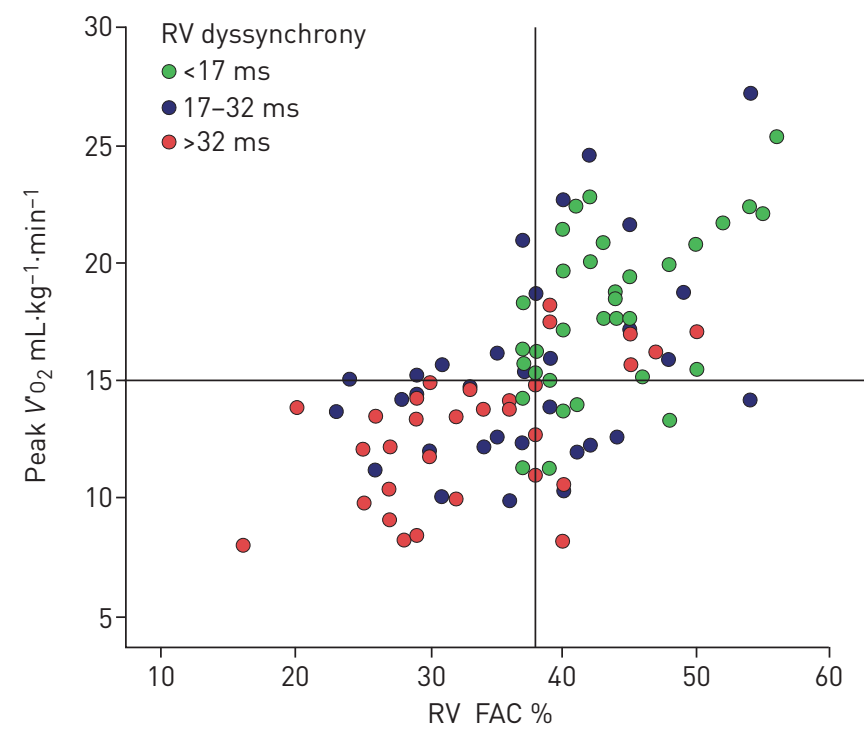

FIGURE 1 Right ventricular (RV) fractional area change (FAC) versus peak oxygen uptake $\left(V^{\prime} \mathrm{O}_{2}\right)$ relationship based by RV dyssynchrony tertiles distribution for the overall population: RV dyssynchrony $<17 \mathrm{~ms}, \mathrm{RV}$ dyssynchrony 17-32 ms and RV dyssynchrony >32 ms.

and TAPSE indicate decreased systolic function and increased dimensions, and thus suggest RV failure as a cause of limitation of maximum cardiac output and exercise capacity. It is of interest that RVFAC was tightly correlated with peak $V^{\prime} \mathrm{O}_{2}$ and clinical/haemodynamic indicators of severity of $\mathrm{PAH}$, further underscoring the importance of preserved systolic function adaptation to afterload in these patients.
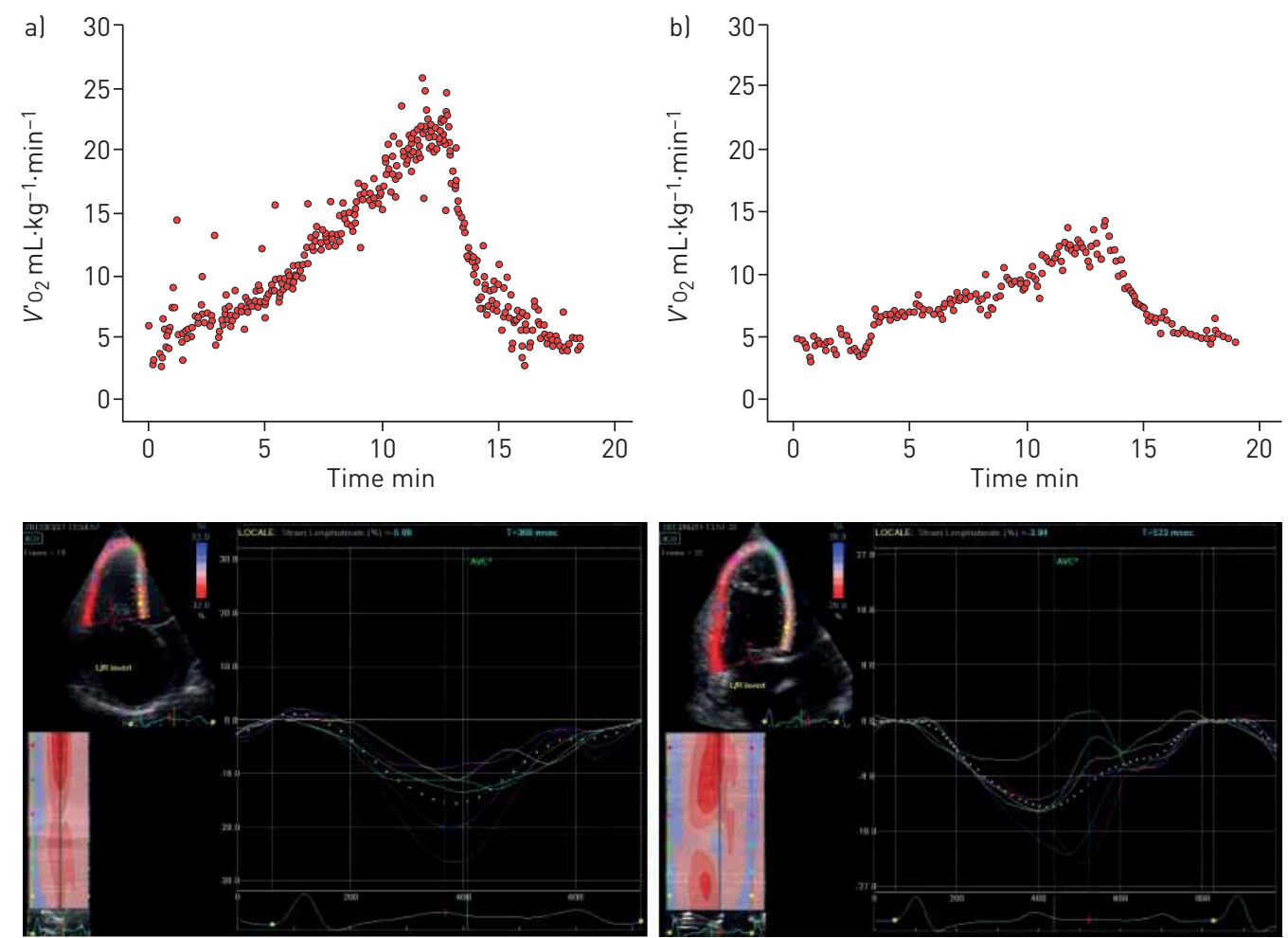

FIGURE 2 Right ventricular (RV) dyssynchrony calculated by the standard deviation of the times to peak-systolic strain for the four mid-basal RV segments (RV-SD4) and the corresponding oxygen uptake $\left(V^{\prime} \mathrm{O}_{2}\right)$ pattern during the cardiopulmonary exercise test, in two different idiopathic pulmonary arterial hypertension patients. a) Good exercise performance with high $V^{\prime} \mathrm{O}_{2}$ peak $\left(23 \mathrm{~mL} \cdot \mathrm{kg}^{-1} \cdot \mathrm{min}^{-1}\right)$ in a patient without significant RV dyssynchrony (RV-SD4: $14 \mathrm{~ms}$ ); b) poor exercise performance with low $V^{\prime} \mathrm{O}_{2}$ peak $\left(12 \mathrm{~mL} \cdot \mathrm{kg}^{-1} \cdot \mathrm{min}^{-1}\right)$ in a patient with significant RV dyssynchrony (RV-SD4: $34 \mathrm{~ms}$ ). 
TABLE 5 Demographic, clinical, haemodynamic, echocardiographic and exercise parameters based on right ventricular dyssynchrony tertile distribution

\begin{tabular}{|c|c|c|c|c|}
\hline & Lower tertile & Intermediate tertile & Upper tertile & p-value \\
\hline Patients & 34 & 32 & 32 & \\
\hline Age years & $54 \pm 12$ & $53 \pm 12$ & $49 \pm 17$ & NS \\
\hline BMI $\mathrm{kg} \cdot \mathrm{m}^{-2}$ & $25.8 \pm 4.4$ & $25.7 \pm 4.2$ & $24.5 \pm 4.6$ & NS \\
\hline WHO class & $2.5 \pm 0.5^{\emptyset}$ & $2.7 \pm 0.4^{+}$ & $3.0 \pm 0.0$ & 0.0001 \\
\hline 6MWT m & $462 \pm 55^{\text {I }}$ & $434 \pm 63^{+}$ & $395 \pm 58$ & 0.0001 \\
\hline \multicolumn{5}{|l|}{ Haemodynamics } \\
\hline mPAP $\mathrm{mmHg}$ & $41.8 \pm 11.2^{1}$ & $44.7 \pm 14.3^{+}$ & $57.7 \pm 15.8$ & 0.0001 \\
\hline RAP mmHg & $6 \pm 2.4^{\pi}$ & $7.5 \pm 3.9$ & $8.9 \pm 5.1$ & 0.009 \\
\hline $\mathrm{Cl} \mathrm{L} \cdot \mathrm{min}^{-1} \cdot \mathrm{m}^{-2}$ & $2.7 \pm 0.3^{\#, 9}$ & $2.4 \pm 0.4$ & $2.3 \pm 0.5$ & 0.0001 \\
\hline PVR Wood units & $6.9 \pm 2.7^{\Uparrow}$ & $8.8 \pm 5^{+}$ & $13.2 \pm 5.1$ & 0.0001 \\
\hline Compliance $\mathrm{mL} \cdot \mathrm{mmHg}^{-1}$ & $1.9 \pm 0.9^{\#, 9}$ & $1.5 \pm 0.6$ & $1.1 \pm 0.5$ & 0.0001 \\
\hline \multicolumn{5}{|l|}{ Echocardiography } \\
\hline $\mathrm{RA}$ area $\mathrm{cm}^{2}$ & $28.4 \pm 7.5^{\Uparrow}$ & $29.4 \pm 9.4$ & $34.5 \pm 11.6$ & 0.021 \\
\hline LV-Eld & $1.2 \pm 0.1^{\#, 9}$ & $1.4 \pm 0.4$ & $1.5 \pm 0.4$ & 0.001 \\
\hline LV-Els & $1.4 \pm 0.2^{\pi}$ & $1.6 \pm 0.4$ & $1.8 \pm 0.9$ & 0.007 \\
\hline RVEDA $\mathrm{cm}^{2}$ & $23 \pm 5.8^{\#, \uparrow}$ & $28.2 \pm 7.8$ & $31.2 \pm 6.1$ & 0.0001 \\
\hline RVESA $\mathrm{cm}^{2}$ & $13 \pm 4^{\# \text { ๆ }}$ & $17.6 \pm 5.7^{+}$ & $21 \pm 5.2$ & 0.0001 \\
\hline RVFAC $\%$ & $43.4 \pm 5.5^{\# .,}$ & $38 \pm 8.5^{+}$ & $32.6 \pm 8.1$ & 0.0001 \\
\hline TAPSE $\mathrm{mm}$ & $21.5 \pm 3.2^{\#, . \Uparrow}$ & $19.1 \pm 3.8$ & $18.1 \pm 3.6$ & 0.0001 \\
\hline \multicolumn{5}{|l|}{ Cardiopulmonary exercise test } \\
\hline $\mathrm{HR}$ rest beats $\cdot \mathrm{min}^{-1}$ & $78 \pm 14^{\pi}$ & $80 \pm 13$ & $86 \pm 15$ & 0.032 \\
\hline HR peak beats $\cdot \min ^{-1}$ & $127 \pm 21$ & $129 \pm 21$ & $129 \pm 29$ & NS \\
\hline$V^{\prime} \mathrm{O}_{2}$ peak $\mathrm{mL} \cdot \mathrm{min}^{-1}$ & $1324 \pm 386^{\#, 9}$ & $1029 \pm 281^{+}$ & $806 \pm 175$ & 0.0001 \\
\hline$V^{\prime} \mathrm{O}_{2}$ peak $\mathrm{mL} \cdot \mathrm{kg}^{-1} \cdot \mathrm{min}^{-1}$ & $17.9 \pm 3.5^{\#, \pi}$ & $15.2 \pm 4.3^{+}$ & $12.9 \pm 2.9$ & 0.0001 \\
\hline$V^{\prime} \mathrm{O}_{2}$ pulse peak $\mathrm{mL} \cdot$ beat $^{-1}$ & $10.8 \pm 2.6^{\#, \pi}$ & $8.0 \pm 1.6^{+}$ & $6.1 \pm 0.9$ & 0.0001 \\
\hline$V^{\prime}$ E peak $L \cdot \min ^{-1}$ & $63.7 \pm 26.7^{\#, 9}$ & $51.8 \pm 13.8$ & $42.6 \pm 10.5$ & 0.0001 \\
\hline$V^{\prime} \mathrm{CO}_{2}$ peak $\mathrm{mL} \cdot \mathrm{min}^{-1}$ & $1354 \pm 459^{\#, 9}$ & $1083 \pm 269^{+}$ & $762 \pm 196$ & 0.0001 \\
\hline $\mathrm{PetCO}_{2}$ peak $\mathrm{mmHg}$ & $25.8 \pm 5.5^{\text {ๆ }}$ & $25.4 \pm 4.9^{+}$ & $21.6 \pm 5.1$ & 0.002 \\
\hline$V^{\prime} E / V^{\prime} \mathrm{CO}_{2}$ slope & $41.7 \pm 10.1^{\pi}$ & $46.8 \pm 13.1$ & $52.1 \pm 15.1$ & 0.006 \\
\hline Work peak W & $80 \pm 36^{\pi}$ & $66 \pm 22$ & $52 \pm 23$ & 0.001 \\
\hline
\end{tabular}

\#: 1 versus $2, p \leqslant 0.04 ;{ }^{9}: 1$ versus $3, p \leqslant 0.03 ;{ }^{+}: 2$ versus $3, p \leqslant 0.03$. BMI: body mass index; WHO: World Health Organization; 6MWT: 6-min walk test; mPAP: mean pulmonary arterial pressure; RAP: mean right atrial pressure; $\mathrm{Cl}$ : cardiac index; PVR: pulmonary vascular resistance; RA area: right atrium area; LV-Eld: left ventricular end-diastolic eccentricity index; LV-Els: left ventricular end-systolic eccentricity index; RVEDA: right ventricular end-diastolic area; RVESA: right ventricular end-systolic area; RVFAC: right ventricular fractional area change; TAPSE: tricuspid annular plane systolic excursion; HR: heart rate; $V^{\prime} \mathrm{O}_{2}$ : oxygen uptake; $V^{\prime} \mathrm{E}$ : minute ventilation; $V^{\prime} \mathrm{CO}_{2}$ : carbon dioxide production; $P_{\mathrm{etCO}}$ : end-tidal $\mathrm{CO}_{2}$ tension.

FIGURE 3 Histograms of oxygen uptake $\left(\mathrm{V}^{\prime} \mathrm{O}_{2}\right)$ peak and 6-min walk distance (6MW) based on right ventricular $(\mathrm{RV})$ dyssynchrony tertiles distribution for the overall population.

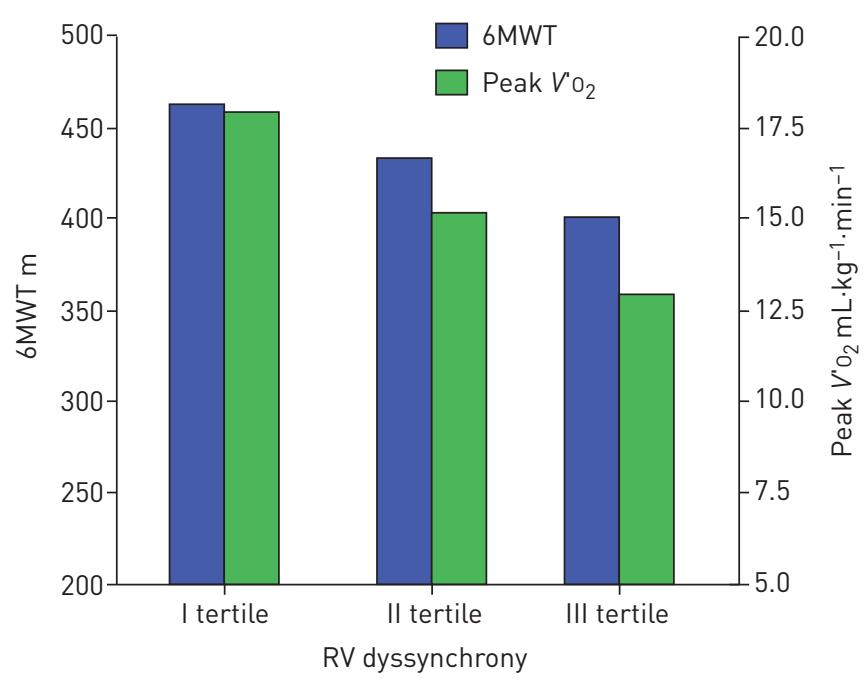




\begin{tabular}{|c|c|c|c|c|c|}
\hline & B & SE & Beta & p-value & $r^{2}$ \\
\hline Haemodynamic model & & & & & 0.48 \\
\hline Intercept & 10.8 & 1.98 & & $<0.001$ & \\
\hline RAP & -0.22 & 0.07 & -0.39 & 0.003 & \\
\hline $\mathrm{Cl}$ & 2.50 & 0.81 & 0.40 & 0.004 & \\
\hline Echo model without RV dyssynchrony & & & & & 0.26 \\
\hline Intercept & 13.1 & 1.57 & & $<0.001$ & \\
\hline RVEDA & -0.14 & 0.06 & -0.31 & 0.02 & \\
\hline LV-Eld & -1.97 & 0.98 & -0.25 & 0.05 & \\
\hline Echo model with RV dyssynchrony & & & & & 0.46 \\
\hline Intercept & 18.5 & 1.85 & & $<0.001$ & \\
\hline RVEDA & -0.15 & 0.05 & -0.36 & 0.02 & \\
\hline LV-Eld & -2.02 & 0.94 & -0.26 & 0.03 & \\
\hline RV dyssynchrony & -0.05 & 0.01 & -0.46 & 0.001 & \\
\hline
\end{tabular}

RAP: mean right atrial pressure; $\mathrm{Cl}$ : cardiac index; RV: right ventricular; RVEDA: right ventricular end-diastolic area; LV-Eld: left ventricular end-diastolic eccentricity index.

In the present study different multivariate analysis models had to be applied to consider the independent impact of physiologically relevant invasive and noninvasive variables on exercise capacity. A single analysis combining right heart catheterisation and echocardiography was not possible owing to the limited number of patients. Both the invasive and the noninvasive approaches capture determinants of RV function. Right atrial pressure is an estimate of RV end-diastolic volume, the cardiac index is the indirect result of RV contractility at any given loading condition, and PA compliance is an estimate of afterload [3]. On the other hand, FAC is a load-dependent estimate of contractility and RA surface a partial and indirect estimate of preload. It is not therefore surprising that the prediction capability of exercise capacity was significant but equally low for both right heart catheterisation and standard comprehensive echocardiography.

RVFAC was chosen over TAPSE for description of the systolic function as it allows a more clear and continuous distribution of patients in respect of increased afterload. In contrast to TAPSE, RVFAC does not present a floor effect in cases of severe RV dysfunction [18] and is not affected by the overall heart motion [19].

Tissue Doppler imaging, speckle tracking echocardiography and also magnetic resonance studies have demonstrated that pulmonary hypertension may be associated with a prolonged RV contraction into LV diastole, resulting in "post-systolic shortening" or asynchrony [5-7], but also with a considerable regional heterogeneity of RV contraction or dyssynchrony [8-12]. Recent studies have shown that RV dyssynchrony is associated with a worse functional state, more severely impaired haemodynamics and echocardiography $[10,11]$, and poor outcome [12]. Correction of dyssynchrony by RV pacing has been shown to restore RV function and cardiac output in rats with monocrotaline-induced PH [20]. Regional heterogeneity is thus an important component of altered RV function in $\mathrm{PH}$. If one assumes that the addition of a measure of regional synchrony of contraction improves the evaluation of RV systolic function, it may not be surprising that it also improves the prediction capability of exercise capacity by echocardiography or right heart catheterisation alone.

Speckle tracking-derived strain of the RV in patients with $\mathrm{PH}$ has been shown to be depressed in proportion to haemodynamic severity and decreased ejection fraction or 6-min walk distance [21] and to be sensitive to therapeutic interventions [21, 22] as well as to the occurrence of cardiovascular events [23]. More recently, this novel imaging modality allowed disclosure of regional differences between apical, mid and base segments which were prominent yet not detected by conventional measures of function such as TAPSE in patients with systemic sclerosis compared with controls [24]. Thus regional inhomogeneity of speckle tracking measurements of RV strain also appears to be a sensitive marker of early or occult myocardial disease. The approach was further developed in the present study with quantification and determination of functional relevance with respect to exercise capacity.

There are several limitations to the present results. First, RV dyssynchrony by 2D speckle tracking was evaluated using software validated for the LV. Whether software specifically developed for the RV might perform better for the prediction of $V^{\prime} \mathrm{O}_{2}$ max is not known. Second, 2D speckle tracking is dependent on plane selection and thus may be methodologically inferior to 3D speckle tracking [10]. However, there has been no 
comparison between these different approaches, and 2D speckle tracking in the present study showed-up as a sensible predictor of exercise capacity. Third, echocardiography and right heart catheterisation were performed at rest only, although exercise measurements would be more pertinent to exercise capacity. However, there are still technical limitations to speckle tracking of the RV during exercise. Fourth, the multivariate analyses had to rely on multiple separate models because of a large number of variables with respect to the patient population. However, idiopathic PAH is an orphan disease and recruiting a much larger population would require a multicentric effort in the long term. Finally, a multiparametric approach combining advanced imaging techniques with invasive assessments would probably improve the characterisation of the functional state, exercise capacity and survival in $\mathrm{PAH}$ [25]. However, this will be for further studies after rigorous identification of all the independent predictors to incorporate in composite scores.

In conclusion, speckle tracking echocardiography of RV dyssynchrony is of added value to right heart catheterisation and standard echocardiography in the evaluation of disease severity of patients with idiopathic PAH.

\section{References}

1 Galiè N, Palazzini M, Manes A. Pulmonary arterial hypertension: from the kingdom of the near-dead to multiple clinical trial meta-analyses. Eur Heart J 2010; 31: 2080-2086.

2 Vonk-Noordegraaf A, Haddad F, Chin KM, et al. Right heart adaptation to pulmonary arterial hypertension: physiology and pathobiology. J Am Coll Cardiol 2013; 62: 25 Suppl, D22-D33.

3 Naeije R. Assessment of right ventricular function in pulmonary hypertension. Curr Hypertens Rep 2015; 17: 35.

4 Rudski LG, Lai WW, Afilalo J, et al. Guidelines for the echocardiographic assessment of the right heart in adults: a report from the American Society of Echocardiography endorsed by the European Association of Echocardiography, a registered branch of the European Society of Cardiology, and the Canadian Society of Echocardiography. J Am Soc Echocardiogr 2010; 23: 685-671.

5 López-Candales A, Dohi K, Bazaz R, et al. Relation of right ventricular free wall mechanical delay to right ventricular dysfunction as determined by tissue Doppler imaging. Am J Cardiol 2005; 96: 602-606.

6 López-Candales A, Dohi K, Rajagopalan N, et al. Right ventricular dyssynchrony in patients with pulmonary hypertension is associated with disease severity and functional class. Cardiovasc Ultrasound 2005; 3: 23.

7 Marcus JT, Gan CT, Zwanenburg JJ, et al. Interventricular mechanical asynchrony in pulmonary arterial hypertension: left-to-right delay in peak shortening is related to right ventricular overload and left ventricular underfilling. J Am Coll Cardiol 2008; 51: 750-757.

8 Kalogeropoulos AP, Georgiopoulou VV, Howell S, et al. Evaluation of right intraventricular dyssynchrony by two-dimensional strain echocardiography in patients with pulmonary arterial hypertension. J Am Soc Echocardiogr 2008; 21: 1028-1034.

9 Meris A, Faletra F, Conca C, et al. Timing and magnitude of regional right ventricular function: a speckle tracking derived strain study of normal subjects and patients with right ventricular dysfunction. J Am Soc Echocardiogr 2010; 23: 823-831.

10 Smith BC, Dobson G, Dawson D, et al. Three-dimensional speckle tracking of the right ventricle: toward optimal quantification of right ventricular dysfunction in pulmonary hypertension. J Am Coll Cardiol 2014; 64: 41-51.

11 Badagliacca R, Poscia R, Pezzuto B, et al. Right ventricular dyssynchrony in idiopathic pulmonary arterial hypertension: determinants and impact on pump function. J Heart Lung Transplant 2015; 34: 381-389.

12 Badagliacca R, Reali M, Poscia R, et al. Right intraventricular dyssynchrony in idiopathic, heritable, and anorexigen-induced pulmonary arterial hypertension: clinical impact and reversibility. JACC Cardiovasc Imaging 2015; 8: 642-652.

13 Galiè N, Humbert M, Vachiery J-L, et al. 2015 ESC/ERS Guidelines for the diagnosis and treatment of pulmonary hypertension. Eur Respir J 2015; 46: 903-975.

14 Sun XG, Hansen JE, Oudiz RJ, et al. Exercise pathophysiology in patients with primary pulmonary hypertension. Circulation 2001; 104: 429-435.

15 Spruijt OA, de Man FS, Groepenhoff $\mathrm{H}$, et al. Effects of exercise on right ventricular contractility and right ventricular-arterial coupling in pulmonary hypertension. Am J Respir Crit Care Med 2015; 191: 1050-1057.

16 Vonk-Noordegraaf A, Westerhof N. Describing right ventricular function. Eur Respir J 2013; 41: 1419-1423.

17 Naeije R, Manes A. The right ventricle in pulmonary arterial hypertension. Eur Respir Rev 2014; 23: 476-487.

18 Mauritz GJ, Kind T, Marcus JT, et al. Progressive changes in right ventricular geometric shortening and long-term survival in pulmonary arterial hypertension. Chest 2012; 141: 935-943.

19 Giusca S, Dambrauskaite V, Scheurwegs C, et al. Deformation imaging describes right ventricular function better than longitudinal displacement of the tricuspid ring. Heart 2010; 96: 281-288.

20 Handoko ML, Lamberts RR, Redout EM, et al. Right ventricular pacing improves right heart function in experimental pulmonary arterial hypertension: a study in the isolated heart. Am J Physiol Heart Circ Physiol 2009; 297: H1752-H1759.

21 Fukuda Y, Tanaka H, Sugiyama D, et al. Utility of right ventricular free wall speckle-tracking strain for evaluation of right ventricular performance in patients with pulmonary hypertension. J Am Soc Echocardiogr 2011; 24: 1101-1108.

22 Badagliacca R, Poscia R, Pezzuto B, et al. Right ventricular remodeling in idiopathic pulmonary arterial hypertension: adaptive versus maladaptive morphology. J Heart Lung Transplant 2015; 34: 395-403.

23 Motoji Y, Tanaka H, Fukuda Y, et al. Efficacy of right ventricular free-wall longitudinal speckle-tracking strain for predicting long-term outcome in patients with pulmonary hypertension. Circ J 2013; 77: 756-763.

24 Mukherjee M, Chung SE, Ton VK, et al. Unique abnormalities in right ventricular longitudinal strain in systemic sclerosis patients. Circ Cardiovasc Imaging 2016; 9. e003792.

25 Vonk-Noordegraaf A, Haddad F, Bogaard HJ, et al. Noninvasive imaging in the assessment of the cardiopulmonary vascular unit. Circulation 2015; 131: 899-913. 